\title{
Trust Based Energy Efficient Relay Selection in Multihop Wireless Network
}

\author{
Shraddha Patil ${ }^{1}$, Pankaj Chandre ${ }^{2}$ \\ ${ }^{1}$ Pune University, PG Scholar, Department of Computer Engineering, Flora Institute of Technology, Pune, Pune-411011, India \\ ${ }^{2}$ Pune University, Assistant Professor, Flora Institute of Technology, Department of Computer Network, Pune-411011, India
}

\begin{abstract}
We will consider the two important aspects of the OR 1. Candidate node selection and 2. Prioritization of the selected nodes in the candidate set. In proposed work we consider the residual energy of the nodes as well as the security parameter of the node by adding a security value to each node at time of deployment of the nodes. We use the secure value (trust) and the residual energy of the node for the candidate selection and the prioritization of the selected relays. Lastly compare proposed system with existing system to demonstrate the results.
\end{abstract}

Keywords: OR, WSN (Wireless Sensor Network), ExOR protocol, Opportunistic routing, Trustworthiness, Residual Energy

\section{Introduction}

Traditional wireless sensor networks were considered as point to point connected nodes by neglecting the broadcast nature of wireless network. Currently opportunistic routing has attracted lot of attention from the researchers in the area of networking. In OR major concern is the candidate selection at the runtime to forward the packet to the destination. Many of the OR protocol has concentrated on the research in the relay selection and neglected the security parameter during selection. Existing work proposed the relay node selection algorithm based on the residual energy of the node. Wireless sensor network (WSN) are everywhere nowadays. In WSNs, thousands of physically embedded sensor nodes are distributed and more modern networks and they used in most applications, it is not possible to change battery each and every time whenever it degrade its value. In order to cooperatively monitor physical or environmental conditions, the main task of sensor nodes is to collect and transmit data. It is well known that transmitting data consumes much more energy than collecting data [2].Energy efficiency for transmitting data, the existing energy-efficient routing protocols are use to to find the minimal energy path between a source and a destination that means a sink to achieve optimal energy consumption [3]-[5].

However, the task of designing an energy-efficient routing protocol, in case of sensor networks, is multifold, wireless sensor network is having multiple functions as its used to balance the distribution energy as well as it used to find out the minimum energy path from a single sensor node to destination, [6].

Furthermore, the unreliable wireless links and network partition may cause packet loss and multiple retransmissions in a preselected good path [7]. Retransmition of selected good packet over the preselected defined path inevitably induces significant energy cost. Therefore, it is necessary to make an appropriate tradeoff between minimum energy consumption and maximum network lifetime.

\section{Related work}

ETX is used by ExOR [1] Extremely opportunistic algorithm to select a candidate forwarder set. It can provide better performances over traditional routing protocols [5]. But there are still some problems in ExOR. After a transmission, all the nodes in the candidate set have to wait for the forwarding of the nodes with higher priority in order. It is not an efficient way to do the spatial reuse. Moreover multicast is not implemented.

MORE [2] randomly mixes packets before forwarding them. This action of forwarding of packets randomness ensures the routers that overhear the same transmission will not forward the same packets. In other words, MORE introduces network coding to OR. MORE support both unicast and multicast. Previously we are using MORE to forword a elected packet to choose relay node. Using ETX in MORE is not suitable because MORE is a versatile scheme, unlike ExOR which is an unfair scheme to use the nodes based on their priorities. It does not need ETX to select candidate nodes with priorities, and treat them based on the different priorities. Moreover, it does not introduce error control and rate control schemes.

SOAR [3] Simple opportunistic adaptive routing protocol was introduced as an advanced to the ExOR protocol in order to support number of various flaws. In SOAR, the candidate forwarders are constrained to be along or near the shortest path from source to destination. A significant difference between ExOR and SOAR is that SOAR performs the routing decision process on a per-packet basis rather than on an entire batch. Computation timing in forworder in the SOAR is simpler than ExOR. And SOAR uses only network paths to calculate shortest distance. Overall, SOAR incurs slightly less overhead than ExOR and restricts flows as close to the shortest path from source to destination as possible. 


\section{International Journal of Science and Research (IJSR) \\ ISSN (Online): 2319-7064}

Index Copernicus Value (2013): 6.14 | Impact Factor (2014): 5.611

\section{Proposed System}

\subsection{Structure}

In proposed system we will implement the candidate selection using residual energy of the nodes and the trust value assigned / calculated by the nodes in the network. We have kept the existing system of residual energy based candidate selection. In our proposed work our aim is to achieve the better results for candidate selection with the improved packet delivery ratio and minimum end to end delay. It consists of three main phases:

1) TBEER selection scheme

2) Phase-2 or Residual energy of nodes

3) Phase-3 or Trustworthiness of nodes

4) Phase- 4 or Candidate selection

5) Phase-5 or Prioritization of Relays

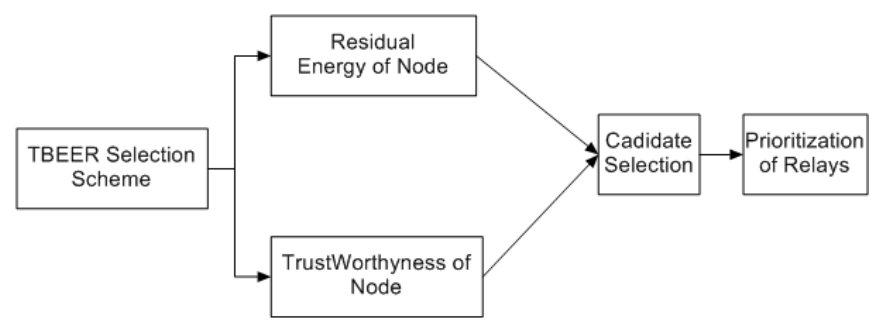

Figure 1: Architecture

\subsection{TBEER selection scheme}

Trust Based Energy Efficient Relay Selection scheme, we are selecting node on trust bas and considering efficiency of energy per node.

\subsection{Residual Energy of nodes}

It is a remaining energy. It is used to describe as a residual energy is the energy which is remaining of something when most of the energy is gone. Energy remains after transmitting the packet and same packet after receiving.

\subsection{Trustworthiness of Nodes}

The name defines worthy of trust. Trustworthy describes something in which you have believe. It is completely reliable. Select the trusted node to whom we can send next packet.

\subsection{Candidate Selection}

Select the candidate after calculating Residual energy and trust parameter of the node.

\subsection{Prioritization of Relays}

From the selected candidate again select one node which is trusted and energy efficient from all other. It should be selected on the base of priority.

\section{Flowchart}

Below is the block diagram of proposed model. Set of rules to be followed by calculation or other problem solving operation. Proposed block diagram description setp by step:

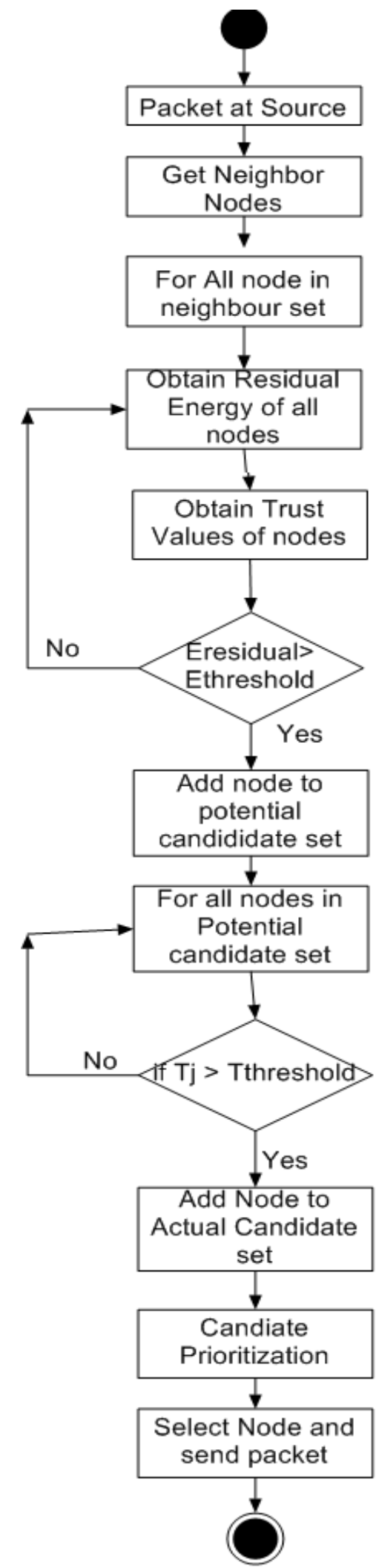

1. We consider a topology having source and destination nodes are fixed. Set of nodes, including source node destination node, neighbor nodes. 


\section{International Journal of Science and Research (IJSR) \\ ISSN (Online): 2319-7064}

Index Copernicus Value (2013): 6.14 | Impact Factor (2014): 5.611

2. Search for neighbor for each node which is present in the topology, collect information about neighbor node.

5. Obtain the Residual energy for all node. To calculate residual energy we have formula which consist of transmitting energy and receiving energy and a constant factor.

6. From the neighbor set of node calculate trust value of each node.

7. Residual energy should be greater than the threshold energy. So we can select the node based on calculated energy.

8. If residual energy of node is greater than the threshold energy then add that node to potential candidate node set. Also check trust value for that node.

9. If trust value is better then add that node to actual set of candidate from the potential candidate node set.

10. Candidate would be selected on the base of priority. From the selected candidate node the node having highest priority will be consider as a best node.

11. Select the best node considering residual energy of the node trustworthiness of the node and priority parameter set on the node.

\section{Selection of Relay Node}

\section{A.Residual Energy of Nodes}

Every node in the network calculates the energy value of each of its neighboring node. If a node has sufficient energy to receive and send the packet to the next hop then only the packet will be forwarded to the next hop. Each node computes the energy of the other nodes using following steps.

1. Calculate the amount of energy consumed by node $\mathrm{C}$ for receiving and forwarding packet.

2. $\operatorname{ER}(\mathrm{c})=\alpha * \mathrm{PR}$

Here $\mathrm{ER}=$ is the amount of energy consumed by node $\mathrm{C}$ to receive all incoming packet.

$\alpha=$ is the configurable parameter (amount of energy required to receive a packet).

$\mathrm{PR}=$ No. of packets received.

3. $\mathrm{ES}(\mathrm{c})=\beta * \mathrm{PS}$.

Here $E s=$ is the amount of energy consumed by node $C$ to send all outgoing packet.

$\beta=$ is the configurable parameter.(amount of energy required to send a packet to next hop).

$\mathrm{Ps}=$ No. of packets sent

4. E Remaining $(\mathrm{c})=\mathrm{E}$ Total $(\mathrm{c})-(\mathrm{ES}(\mathrm{c})+\mathrm{ER}(\mathrm{c}))$

Using above steps we calculate the remaining energy of the nodes in the network. The nodes in the network can take part in candidate selection only if they have sufficient energy to transmit the packet to the next hop.

\section{B. Trustworthiness of node:}

Every node in the network calculates the trustworthiness of the neighboring node using the following equation. Trust value of a node is calculated by using following equation,

$$
\operatorname{Ti}(\mathrm{ji}, \mathrm{n})=\mathrm{Rji}(\mathrm{n}) / \mathrm{Fji}(\mathrm{n})
$$

Ti $(\mathrm{j}, \mathrm{n})=$ Trust value of node $\mathrm{i}$ assigned/calculated by node $\mathrm{j}$ during nth topology cycle. Where Rji(n) and Fji (n) are the number of packet that have been received by $j$ and forwarded from $\mathrm{i}$ at time $\mathrm{t}$ respectively, and $0 \leq \mathrm{T}(\mathrm{j}, \mathrm{t}) \leq 1$.

Trust value of a node is updated after every topology change using following mathematical equation.

$$
\operatorname{Ti}(\mathrm{j}, \mathrm{n})=\alpha . \operatorname{Ti}(\mathrm{j}, \mathrm{n}-1)+(1-\alpha) \cdot \operatorname{Ti}(\mathrm{j}, \mathrm{n})
$$

Where, $\operatorname{Ti}(\mathrm{j}, \mathrm{n})$ is node i's trust value measured during $\mathrm{nth}$ topology updating cycle. $0<\alpha<1$ is a weighting factor used to tradeoff between current measurement and previous estimation.

The calculated trust value is then used for the candidate selection and prioritization of the relays.

\section{Candidate Selection Algorithm}

We a new algorithm for the candidate selection in multi-hop wireless network. The proposed candidate selection algorithm based on the residual energy of the nodes in the network and thrust value of the nodes in the network. Proposed candidate selection algorithm performs the candidate selection in efficient and effective way to achieve the better protocol performance.

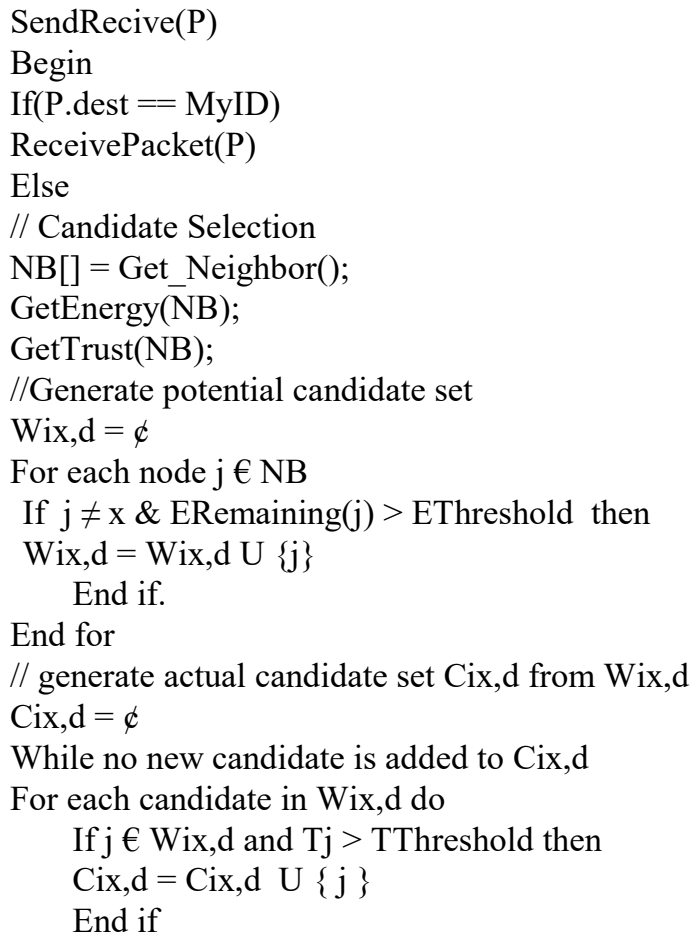

End for

End While

//Candidate selection end

// Prioritization

Sort Node in Cix, $\mathrm{d}$ according to decreasing values of their trust.

// Prioritization

Add sorted Cix,d to the packet.

Send $(\mathrm{P})$;

End else

$$
\text { End begin }
$$




\section{International Journal of Science and Research (IJSR) \\ ISSN (Online): 2319-7064}

Index Copernicus Value (2013): 6.14 | Impact Factor (2014): 5.611

In this section, we will describe how to select and prioritize the forwarder set using residual optimal energy strategy on each node and the trustworthiness of each selected node, also we check how to choose the optimal relay node among potential forwarders that respond in a priority order. So basically residual energy optimal energy trustworthiness parameter and priority will use to select the node In addition, the transmitted data can be naturally classified into two categories:

1) The former is the collected data of its own; and 2) the latter is the relay data from other nodes. We introduce ENS_OR algorithm for energy saving to select the next relay node which has the highest priority in forwarder set to forward the incoming ENS_OR algorithm. Below algorithm describes the pseudocode of ENS_OR algorithm.

\section{Conclusion}

Wireless sensor network (WSN) offers a wide range of applications in areas such as traffic monitoring, medical care, inhospitable terrain, robotic exploration, and agriculture surveillance In this paper we will implement the candidate selection using residual energy of the nodes and the trust value assigned / calculated by the nodes in the network. We have kept the existing system of residual energy based candidate selection. In our proposed work our aim is to achieve the better results for candidate selection with the improved packet delivery ratio and minimum end to end delay

\section{References}

[1] Juan Luo, Member, IEEE, Jinyu Hu, Di Wu, Member, IEEE, and Renfa Li, Senior Member, IEEE ,'Opportunistic Routing Algorithm for Relay Node Selection in Wireless Sensor Networks", IEEE TRANSACTIONS ON INDUSTRIAL INFORMATICS, VOL. 11, NO. 1, FEBRUARY 2015

[2] VVangBo HuangChuanhe YangVVenzhong VVangTong ," Trust Opportunistic Routing Protocol in Multi-hop Wireless Networks", 978-1-4244-58493/10/\$26.00 C2010 IEEE.

[3] D. Bruckner, C. Picus, R. Velik,W. Herzner, and G. Zucker, "Hierarchical semantic processing architecture for smart sensors in surveillance networks," IEEE Trans. Ind. Informat., vol. 8, no. 2, pp. 291-301, May 2012.

[4] G. J. Pottie and W. J. Kaiser, "Wireless integrated network sensors," Commun. Assoc. Comput. Mach., vol. 43, no. 5, pp. 51-58, 2000.

[5] L. LoBello and E. Toscano, "An adaptive approach to topology management in large and dense real-time wireless sensor networks," IEEE Trans. Ind. Informat., vol. 5, no. 3, pp. 314-324, Aug. 2009.

[6] D. Hoang, P. Yadav, R. Kumar, and S. Panda, "Realtime implementation of a harmony search algorithmbased clustering protocol for energy efficient wireless sensor networks," IEEE Trans. Ind. Informat., vol. 10, no. 1, pp. 774-783, Feb. 2014.
[7] D. Zhang, G. Li, K. Zheng, X. Ming, and Z.-H. Pan, “An energy-balanced routing method based on forward-aware factor for wireless sensor networks," IEEE Trans. Ind. Informat., vol. 10, no. 1, pp. 766-773, Feb. 2014.

[8] F. Ren, J. Zhang, T. He, C. Lin, and S. K. Ren, "EBRP: Energybalanced routing protocol for data gathering in wireless sensor networks," IEEE Trans. Parallel Distrib. Syst., vol. 22, no. 12, pp. 2108-2125, Dec. 2011.

[9] Behnad and S. Nader-Esfahani, "On the statistics of MFR routing in one-dimensional ad hoc networks," IEEE Trans. Veh. Technol., vol. 60, no. 7, pp. 32763289, Sep. 2011.

[10] Ghasemi and S. Nader-Esfahani, "Exact probability of connectivity one-dimensional ad hoc wireless networks," IEEE Commun. Lett., vol. 10, no. 4, pp. 251-253, Apr. 2006 . 